\title{
A Multi Logistics Network Optimization Model of Power System based on Stochastic Demand and Inventory Decision
}

\author{
Youpeng Zhang, Jibin Zhu, Yunxin Chang, Yonghao Guo, Chaofeng Zhu \\ PINGGAO GROUP CO.,LTD, Pingdingshan 467001, Henan, China
}

Received: April 8, 2021. Revised: August 10, 2021. Accepted: August 23, 2021. Published: August 25, 2021.

\begin{abstract}
According to the disposal demand for packets and all kinds of constraints in the current condition, this paper discusses a modeling method of extended doublelayer capacitated arc routing problems with Capacitated Arc Routing Problem (CARP) optimization model based on express logistics. The model is described in detail, and the solution of the model is discussed. The paper analyses and discusses the solution to complexity of the model, and proposes a better solution to the double-layer CARP optimization model. According to the scheme, the paper chooses a sort of improved ant colony algorithm to solve the model. And the results show that the scheme is beneficial to controlling the cost of logistics links, to minimizing purchase cost, transportation cost and delivery time. The scheme plays a very imperative role in enhancing the competitiveness of enterprises.
\end{abstract}

Keywords-Multi Logistics Network, Power Logistics, Power System, Stochastic Demand, Inventory Decision

\section{INTRODUCTION}

L ogistics is the system designed for the realization of rationalization, automation and mechanization in logistics process. Its purpose is to be effective in space and time. Modern logistics not only involves the procedure about the goods distribution from producers to consumers, but also involves the deals between producers and manufactures, and the transportation, storage as well as information of producers.

A delivery and pickup supply chain system that consists of one supplier, several retailers, and multiple disposal facilities is considered in this study. The main objective of this study is to minimize overall cost. In the present study, retailers play roles in distribution and collection points. At present, scholars have further research in various links of modern logistics. In the logistics system modeling, the Petri network model was chosen. Kelleret proposed general framework of object-oriented flexible manufacturing system simulation model [1], and Anglani made the conversion from conceptual model to practical model through the use of object-oriented technology to construct development environment of flexible manufacturing system simulation model [2]. After introducing the concept of object-oriented to the discrete event system simulation and modeling, Wolfgang et.al [3] proposed the system framework---Beta SIM. As for the supply of logistics, Ganapathy et.al solved decision-making problem of complex supply chain logistics planning by use of simulation. When Bruzzone et.al [4] evaluated performance of supply chain logistics system, they used the method and technology of artificial intelligence and simulation. In the literature [5], Tanigichi's team proposed the integrated model of dynamic traffic simulation and vehicle routing problem with time windows. Bruzzone designed decision supporting system composed by dynamic database, heuristic rules, and dynamic process simulation modules concerning with optimization module [6]. Fu built a simple stochastic optimization model for inventory in literature [7].

CARP (Capacitated Arc Routing Problem) with delivery or pickup is typically considered for the past three decades. Literature [8] addressed the problem to maximize system profit. The problem was formulated and a Lagrangian-based approach was designed. Literature [9] considered a delivery problem with one depot and several retailers. Literature [10] considered a problem in which a system comprised multiple suppliers and one assembly plant. For more details on CARP with delivery or pickup, interested readers can refer to Literature [11] and Literature [12]. CARP with both delivery and pickup has been studied in only two articles. Literature [13] addressed a production-CARP with delivery and pickup.

Each retailer faces deterministic demand on each time unit, and each disposal facility faces disposal and holding costs. We assume that returned items that need to be picked up reach each retailer. If a retailer is served, then the delivered quantity allows maximum inventory level to be reached. Returned items should be picked up simultaneously. Otherwise, these items are stored at the retailer, and hence, holding cost occurs. Moreover, items picked up for disposal facilities are stored and disposed of when a certain inventory level is reached. The main objective of the present study is to find a replenishment policy for each retailer and a distribution policy to minimize total cost. We assume that vehicles arrive at each node immediately after returned items. Then, after serving all the retailers on its route, the vehicle returns to the nearest disposal facility. Although the scholars of various countries have made many achievements in the field of modern logistics, many kinds of research methods have not solved the practical problems in large scale case. And in the 
scheduling and controlling of logistics system, there is large space for improvement [14]. According to solving the practical problems of the big logistics system, the paper proposes the method of modeling, analysis, and studying for extended double-layer CARP.

\section{THE EXTENDED DOUBLE-LAYER CARP MODEL}

Express logistics consists of carrier, transportation, collection and distribution. The mathematical modeling method of logistics system is the basis for vehicle scheduling, path optimization, and rapid logistics management. This paper will express logistics optimization problem into optimization problem of CARP. Based on the standard CARP model, the paper establishes an extended double-layer CARP model. This model is usually only for one city. Considering with the problems of macro allocation of logistics (distribution center location and quantity), the model can complete the related services as soon as well, such as determining the shortest route of the vehicle in order to provide timely services to customers.

Double-layer CARP optimization problem can be abstractly described: In a given directed graph, each arch has an empty vehicle costs; there is a penalty term between any two adjacent steering arcs segments; the service costs and demand quantity of arbitrary arcs need to provide services are known; delivery vehicles served for each arc are identical, namely speed, load and acquisition cost are the same; repeatedly perform the same task in a given period; the number of delivery vehicles, the number and location of distribution centers are decision variables; all raised the demand for services within the arc of a service must obtain the corresponding period services; when performing a single task, the total volume of services each delivery trucks accept cannot outstrip its maximum load; the consumption of time of each delivery vehicles must be within the maximum service time; After the tasks are executed, all delivery vehicles must return to their starting distribution center; some services are banned; from the micro level, in order to serve for arcs demand for services, optimal driving route need to be developed; from macro level, the optimal number of vehicles need to be determined, and the number and location of distribution centers need to be determined; and finally, the purpose of the double-layer optimization is to minimize the running costs and fixed costs.

\section{A. The Input Variables}

In this paper, the streets, crossroads, and the ends of the road sections are simplified as nodes. The roads and streets are simplified as arcs. Thus, the entire network can be simplified as an undirected graph $G=(V, E)$. E represents arcs set, and $V$ represents point set. When vehicles are passing through arc segments $u \in A$, the transportation cost is $s(u)$, and the emptyload cost is $d(u)$. The empty-load cost refers to costs made by vehicles do not load any goods through a section of road. If transportation costs related to vehicle load and arc length, and empty cost only relates to the latter, we can obtained the following formula (1) and (2),

$$
\begin{gathered}
d(u)=\alpha \times \operatorname{Dis}(u) \\
s(u)=\beta \times \operatorname{Dis}(u) \times q(u)
\end{gathered}
$$

In the formula (1) and (2), $\beta$ is transportation cost coefficient, $\alpha$ is empty-load cost coefficient, $\operatorname{Dis}(u)$ is arc length, and $q(u)$ is the vehicle load. Coordinates of the start point of arc $u$ is $\left(x_{1}, y_{1}\right)$, and the coordinates of the end point is $\left(x_{2}, y_{2}\right)$ . Thus, the arc length can be expressed as formula (3).

$$
\operatorname{Dis}(u)=\sqrt{\left(x_{1}-x_{2}\right)^{2}+\left(y_{1}-y_{2}\right)^{2}}
$$

Concerned that there are many kinds of materials in each distribution center, and the materials are different in the specifications, cost, and quantity. The paper assumes that $D e=\left\{D_{1}, D_{2}, \cdots, D_{\left(N_{1}\right)}\right\}$ is distribution center set, and the coordinate of distribution center $i$ is $D_{1}=\left\{x_{i}, y_{i}\right\}$. The number of distribution center is $N_{1}$. The number of materials $j$ belong to distribution center $i$ is $S_{i j}, C_{i j}$ is the material cost.

In reality, load, volume, and cost of each vehicle are not the same. For the convenience of study, the paper does not take these aspects of vehicle (including the driver, vehicle arrangement, gas, repair, volume) into consideration at the same time. In addition, the paper hypothesizes there is no difference in load among various vehicle. $p_{i}$ represents that are controlled by distribution centers $i$, then $P=\left\{p_{1}, p_{2}, \cdots, p_{\left(N_{1}\right)}\right\}$ represents the specific distribution of vehicles that are controlled by distribution center. $q_{i}$ is used to represent the cost of vehicles that are controlled by the $i$ th distribution center, then $Q=\left\{q_{1}, q_{2}, \cdots, q_{\left(N_{1}\right)}\right\}$ represents the cost of vehicles that are controlled by all distribution centers, and the cost is simplified as a fixed cost. All vehicles load are assumed as $W$.

For the convenience of study, the paper only takes weight and quantity of materials into consideration. And the paper overlooks other factors, such as the mixed-up, volume, loading and unloading of materials etc. In addition, materials in different sizes are regarded as various materials. The number of types of materials is assumed as $N_{2}$, and the demand for materials $j$ by node $i$ is $K_{i j}$.

The input variables can be summarized that an undirected graph $G=(V, E), E$ represents arc set, and $V$ represents point set. The transportation cost of each arch is $s(u)$. The empty-load cost of vehicles is $d(u)$. The distribution center set is $D_{e}=\left\{D_{1}, D_{2}, \cdots, D_{\left(N_{1}\right)}\right\} \cdot N_{1}$ represents the number of distribution centers. $C_{i j}$ and $S_{i j}$ represents materials cost and number of distribution center $i$ separately. The number of materials' types is $N_{2}$, and the demand for materials $j$ by 
node $i$ is $K_{i j}$. The specific layout of the vehicles controlled by all distribution centers is $P=\left\{p_{1}, p_{2}, \cdots, p_{\left(N_{1}\right)}\right\}$; And the cost of the vehicles is $Q=\left\{q_{1}, q_{2}, \cdots, q_{\left(N_{1}\right)}\right\}$; The load of all vehicles is $W$.

\section{B. The Output Variables}

The variables can be represented by formula (4),

$$
R_{i j}=\left(a_{i j}, b_{i j}, c_{i j}, d_{i j}, e_{i j}, f_{i j}\right)
$$

In the formula (4), $R_{i j}$ represents the $j$ th routing of vehicle $i, a_{i j}$ represents the start node of the routing, $b_{i j}$ represents the distribution and storage center for the goods transported by the routing, $c_{i j}$ represents the purpose node of the routing, $d_{i j}$ is the whole weight of the goods, $e_{i j}$ is the total cost of the routing for the delivery of goods, and the $f_{i j}$ is the consumption of time for the delivery of goods.

\section{The Object Function}

Express logistics optimization is a multi-objective optimization problem, which has two main objectives to minimize delivery time and the transportation material cost. The object function for minimizing the delivery time can be expressed as formula (5).

$$
\text { Min Time }=\max _{i}\left(\sum_{j} f_{i j}\right)
$$

In addition, minimizing the purchase and transportation cost is to minimize the total cost for material transportation. The total cost refers to the sum of transportation cost and purchase cost. The purchase cost can be expressed as formula (6).

$$
C_{a}=\sum_{i} e_{i}
$$

The transportation cost can be expressed as formula (7).

$$
C_{B}=\sum_{i} q_{i} \delta_{i}+\sum_{i}\left\{\alpha \times\left\langle a_{i} b_{i}\right\rangle+\alpha \times\left\langle b_{i} c_{i}\right\rangle+\beta \times\left\langle b_{i} c_{i}\right\rangle \times d_{i}\right\}
$$

In the formula (9), $d_{i}$ is a Boolean variable, which represents the condition whether the vehicle $i$ participated the transportation of materials. $\left\langle a_{i} b_{i}\right\rangle$ represents the distance between $a_{i}$ and $b_{i}$, and $\left\langle b_{i} c_{i}\right\rangle$ represents the distance between $b_{i}$ and $c_{i}$. According to the explanation mentioned above, the optimization object function for the transportation cost of materials is

$$
\operatorname{Min} C_{T}=C_{A}+C_{B}
$$

\section{The Constraint Condition}

(1)The overloading of vehicles is not allowed. Because the overloading of vehicles is a serious threat to the safety of vehicles and drivers, and the time consumption cannot be ensured when transporting supplies to designated location, so the paper forbids every vehicles no to overload in express logistics problems. In the feasible scheme of express logistics problem, for all $i$ and $j, d_{i j} \leq W$. (2)In the practical operation, for the convenience of management, vehicles are ordered to come back to the distribution center for their parking. This paper makes all vehicles return to their own distribution centers after the completion of tasks. (3) The demand for materials by all arcs should be met. (4) The load of all materials onto all vehicles should be completed in one distribution center in single transportation. (5) The number of vehicles sent by a distribution center should not outstrip the total number of vehicles in it. And the number of vehicles which centers are allowed to send is exactly which they owned.

\section{THE SOLUTION COMPLEXITY ANALYSIS FOR EXTENDED DOUBLE-LAYER CARP MODEL}

\section{A. The Main Problems}

The main problems for the solution to double-layer CARP optimization model are the macro allocation and the micro path optimization. In the first problem, the paper limits the number of candidate distribution centers in each feasible scheme to be less than or equal to $c$. The number of nodes is $N$, and then available schemes can be expressed as formula (9).

$$
\sum_{k=1}^{c} C_{N}^{k}
$$

In the second problem, assuming the number of the distribution centers is $b$, then available schemes can be expressed as formula (10).

$$
b^{a} \prod_{i=1}^{a} Q_{i}
$$

Therefore, the number of available schemes for double-layer CARP optimization is

$$
\sum_{k=1}^{c}\left(k^{a} C_{N}^{k} \prod_{i=1}^{a} Q_{i}\right)
$$

At the same time, the solution to the problem is apparently more difficult than to the multi-distribution CARP problem. Double-layer CARP problem is also a sort of NP-Hard problem.

Though the following simple arithmetical example, the solution complexity for double-layer CARP problem can be explained effectively. In this case, the total number of arcs and nodes are respectively 9 and 6 , which named by the serial number of arcs and nodes respectively, such as arc 1, node 1etc.. As shown in Figure 1, the demand (in kilograms), service cost (in RMB), empty-load cost (in RMB) and the serial number of each arc are given.According to amount of the arc demand, whether the arc should provide services can be determined. If demand quantity is less than 0 , the arc does not need to provide services; conversely, it needs to. Furthermore, Figure 1 also marked the turning penalty (in RMB) for two adjacent arcs in addition to the value of 0 . We can do the following assumptions in the calculation example: construction expenditure for each warehouse is $10000 \mathrm{RMB}$; the purchase cost of vehicles is 2000 
RMB; the speed and the capacity of the vehicles are limited to $100 \mathrm{~km} / \mathrm{h}$ and $15 \mathrm{~kg}$ respectively; the longest service time is 0.5 hours; all of the tasks will be repeated for 100 times in three years; the conversion coefficient of driving distance and emptyload cost is 1.6.

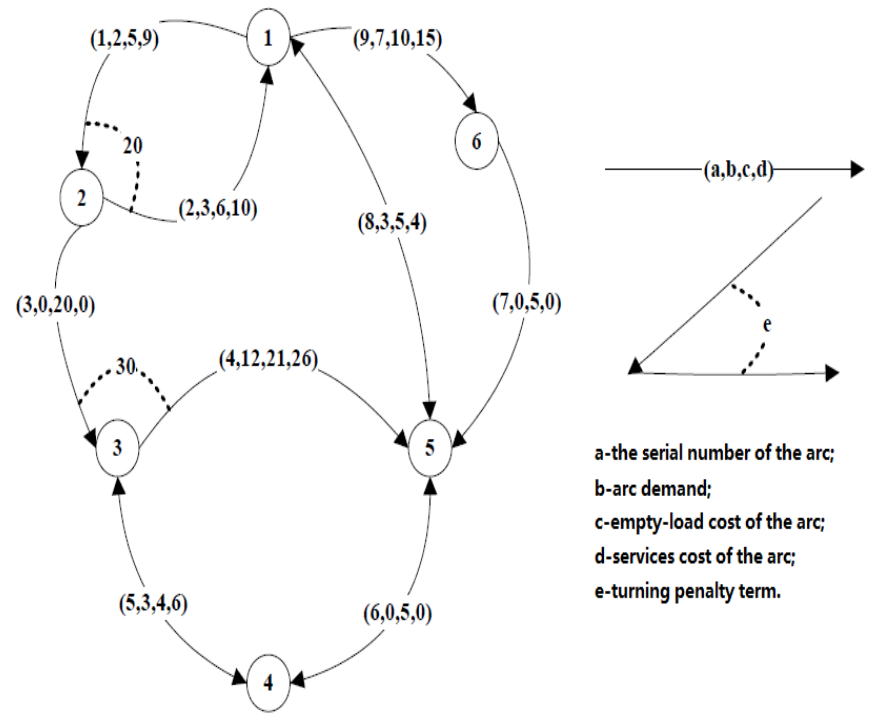

Fig. 1. The arithmetical example for the double-layer CARP optimization problem

In order to solve the double-layer CARP optimization problem shown in figure 1, there are two problems need to be solved. The first one is the macro allocation problem, and the second one is the micro path optimization problem. In the first problem, the number of candidate distribution centers in each feasible solution is limited to less than or equal to 3 , the number of nodes is 6 , then there are $\sum_{k=1}^{3} C_{6}^{k}=6+15+20=41$ available schemes for the problem. In the second problem, we assume that there is only one given distribution center, then there are $34^{3}=39304$ available schemes for the problem. Supposed the number of distribution center is 2 , then the number of available alternatives in micro path problem is $(2 \times 34)^{3}=314432$. Supposed the number of distribution center was 3 , then the number is $(3 \times 34)^{3}=1061208$. Taking the in macro configuration optimization problems into consideration, the number of available schemes in this double-layer optimization arithmetical example is $6 \times 34^{3}+15 \times(2 \times 34)^{3}+21 \times(3 \times 34)^{3}=27237672$

According to the results mentioned above, the number of available schemes provided by double-layer CARP optimization algorithm is 87 times as multi-distribution center CARP optimization algorithm, and is 693 times as standard CARP optimization. According to the analysis mentioned above, the number of alternative distribution centers plays an imperative role in the solution to the double-layer CARP optimization. The greater the number is, the more complex the solution to the problem is. In the practical logistics work, large logistics distribution centers are affected by many factors in a city. Thus, this paper proposes comprehensive evaluation method to rank the location of distribution centers so as to reduce the number of alternative distribution centers in double- layer CARP optimization problems. Then reduce the complexity of the algorithm and accelerate the completion of solution.

\section{B. Integrated Solution Framework of Double-Layer CARP}

The integrated solution framework of double-layer CARP is shown in Figure 2. Firstly evaluate and rank alternative express logistics distribution centers in comprehensive way, and propose the feasible solutions to macro configuration optimization problem based on it. Then, with the constraint of the criteria of feasible solutions to the problem, propose feasible solution according to the route optimization problem. Concern with macro configuration optimization problem and micro allocation optimization problem and solve them simultaneously. In the integrated framework, evaluation is only need for integrated scheme of double-layer optimization problem based on express logistics system. Through iteration in many times, a better solution to the optimization problem will be get.

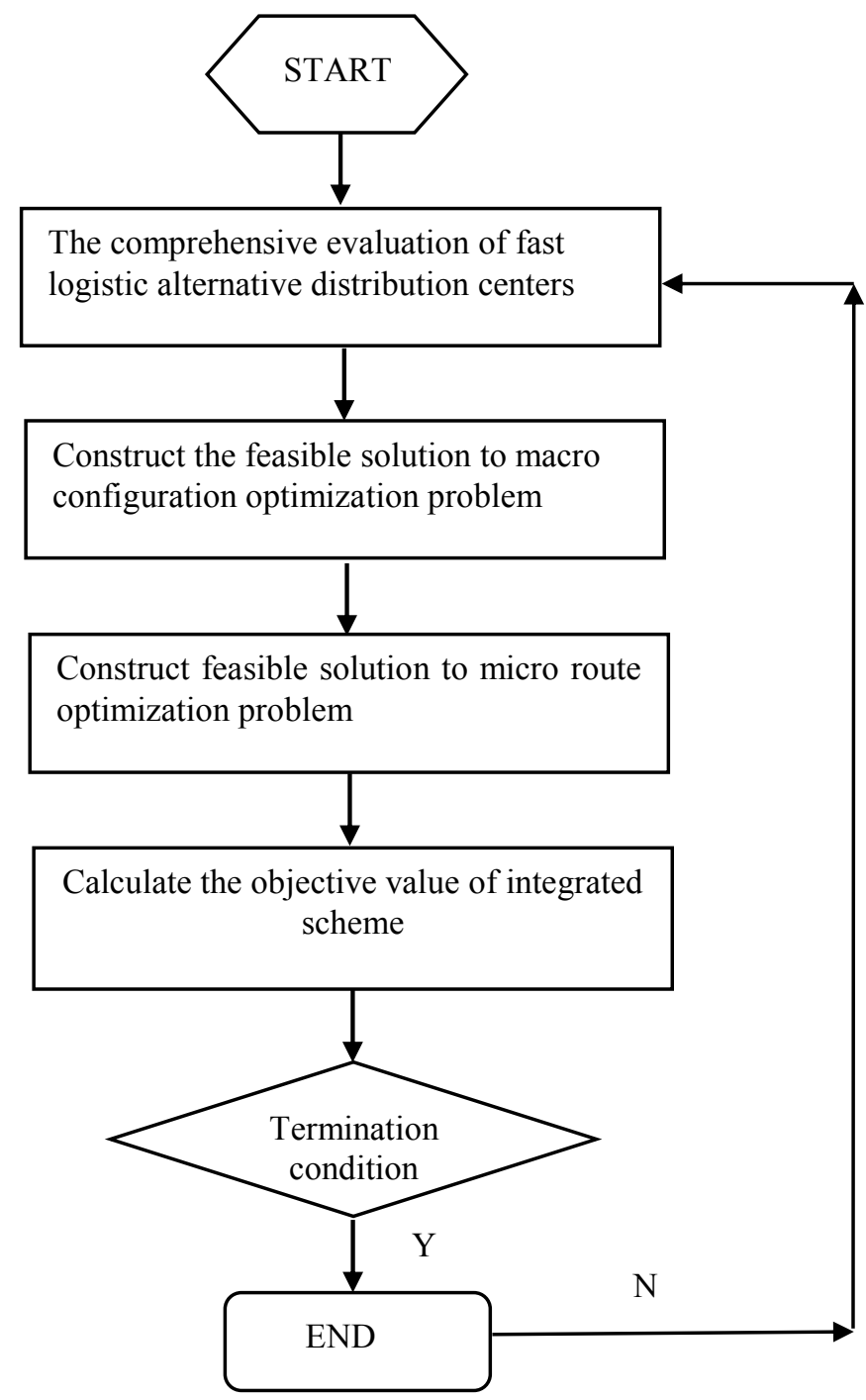

Fig. 2. Overall framework for solving express logistics system level optimization problem 


\section{Hierarchical Solution Framework of Double-layer}

\section{CARP}

As shown in Figure 3, in hierarchical solution framework, solve the macro configuration optimization problems firstly, then deal with the micro route optimization problems.

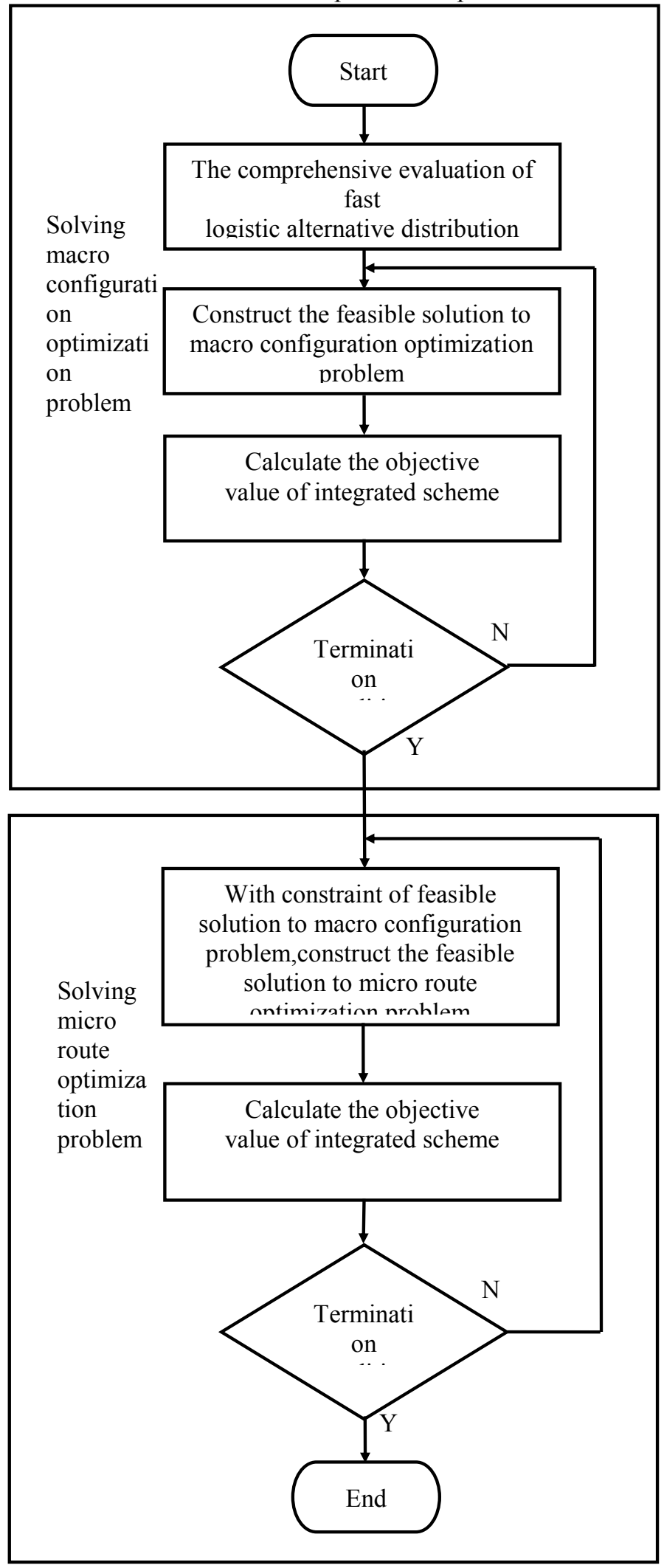

Fig.3. Hierarchical frameworks for double-layer optimization problem in the logistics system

When solving the first problem, construct feasible schemes for this problem at first; and then calculate their objective values in order to evaluate these schemes in an effective way; finally, calculate the best scheme for the problem through iteration in many times. As for the second optimization problems, firstly, construct the feasible schemes with the constraint of the best scheme for the first problem; then evaluate the problem; finally, calculate the best scheme for the problem through iteration in many times. The integration of two schemes is the optimized scheme for the whole double-layer optimization problem. In general, when solving these two kinds of optimization problems in hierarchical framework, setting up two types of termination conditions is necessary, termination conditions are shown in Figure 3.

\section{EXPERIMENTAL ANALYSIS}

In the double-layer optimization problems of express logistics system, there are many available macro configuration optimization schemes; accordingly, there are also many available micro route optimization schemes. In the integrated solution framework, solve the two kinds of optimization problems at the same time. Search the solution space with the sum of both schemes; if the best scheme is needed, the condition in which both schemes are the optimized schemes must be met. The better scheme is hard to get in the Optimization problem of logistics system in the integrated solution framework in logistic system double-layer optimization problem. However, it can be obtained under hierarchical solution framework condition, because two kinds of optimization problems can be solved in the framework respectively, and through integration of both schemes in an effective way, the optimized scheme can be obtained. Therefore, in this paper the hierarchical solution framework is chosen for solving double-layer optimization problem in logistics system..

In order to test the optimization effect of double-layer optimization model, this paper chooses an improved ant colony algorithm according to the network structure shown in Figure 4 for the optimization calculation[14].

In the road network, assuming there are 100 nodes (nodes are labeled 1-100) and 360 directional arcs. In Figure 4, five different types of line segment symbol are defined. The demand of each arc (in tons), service cost (in dollars) and empty-load cost (in dollars) can be marked in the graph through the five types of line segment symbol. 


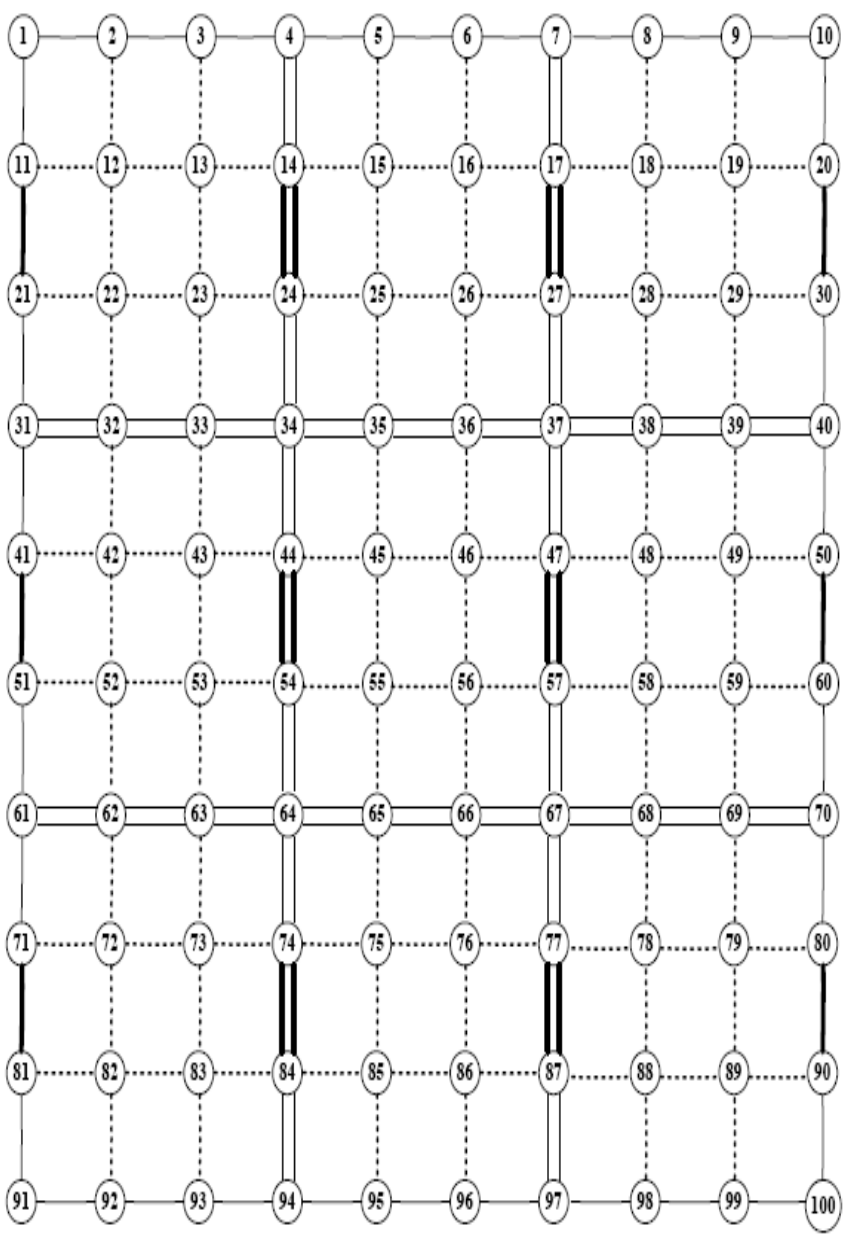

Fig. 4. Road network structure

In this paper, a pair of unidirectional opposed arcs is used to depict a bidirectional arc. In order to distinguish various arcs, each arc is defined by corresponding type attribute. Whether the arc is a unidirectional one or a bidirectional one can be distinguished through it. If the property value is greater than 0 , it is a bidirectional one. If it is equal to 0 , the unidirectional one. If property values of both arcs are equal and greater than 0 , they are two arcs corresponding to a bidirectional arc in different direction.

In the application algorithm of this paper[15], some values are defines as follows.

a) all turn penalty item as 0 ;

b) The construction cost of each distribution center is 10 million $\mathrm{RMB}$, and the purchase cost of each delivery vehicle is 200000 RMB;

c) The load and speed of each delivery vehicle are respectively 11 tons and 120 kilometers.

d) The conversion coefficient between distance and empty-load cost is 2 ;

e) The longest time within each service period is 1 hours.

f) The same task will be operated for 10000 times within certain period.

The improved ant colony optimization method is used to solve the examples in this paper, the optimization results are shown below.

The number of distribution centers is (located at node 34), and the construction cost of the center is 10 million RMB. The number of each distribution vehicles is 19 , and the purchase cost is 3.8 million RMB;Each route starts from node 34 and ends in node 34 . In this example, the single time of service cost (the sum of empty-load cost and service cost) is 990 RMB. Because the same task will be repeated for 10000 times, the operation cost of this example is 9.9 million RMB. The objective value(the sum of fixed cost and operation cost) of the optimization results (the optimized scheme) is 23.7 million RMB.

Furthermore, the improved ant colony optimization method was used to analyze the optimal result's affect which causes by the cost of the distribution centers. During solving this CARP model the cost of building distribution centers were set to 100,000 yuan, 500,000 yuan and 100 yuan, five million yuan and 10 million yuan, the other conditions remain unchanged. The optimization results are shown in Figure 5 and Figure 6.

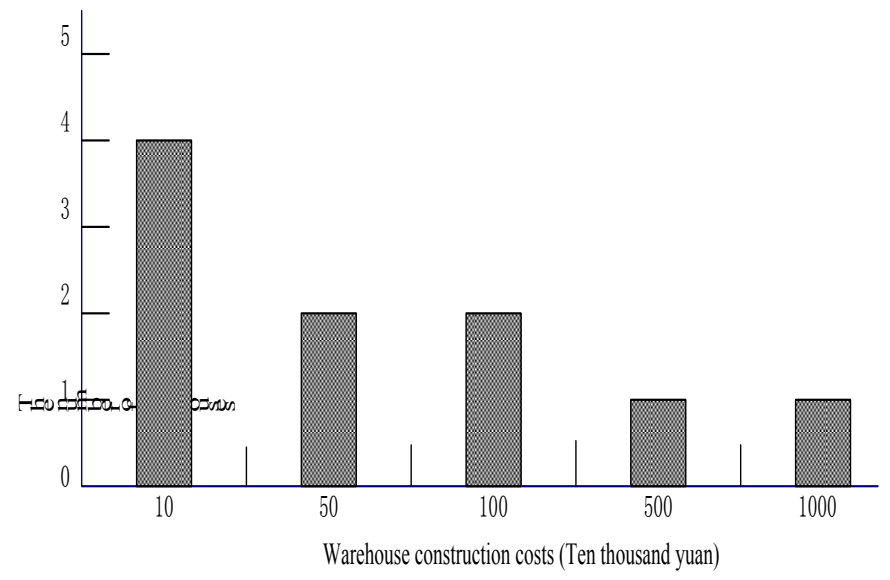

Fig.5. The relationship between warehouse number and costs

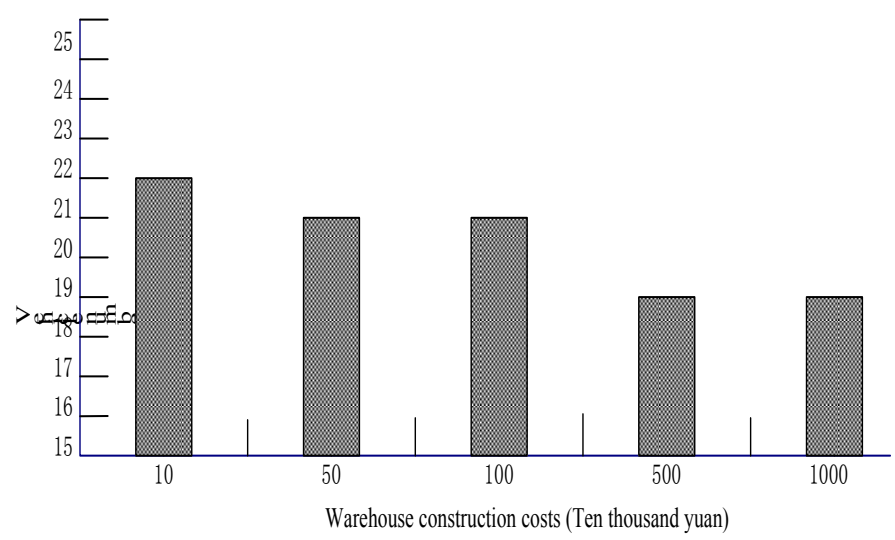

Fig. 6. The relationship between warehouse construction costs and vehicle number

From Figure 5 it can be seen, the warehouse number of distribution center is inversely proportional to the construction cost of distribution center. If the construction cost of distribution center is 10 million $\mathrm{RMB}$, then just build a distribution center is undoubtedly a best choice; if the construction cost of distribution center is 100 thousand yuan, and build 4 distribution centers is a optimal selection. From Figure 6 it can be seen, the warehouse construction cost of distribution center is nothing with the number of vehicles. 


\section{CONCLUSION}

The paper discusses a sort of modeling method for an innovative extended double-layer CARP optimized model based on express logistics, analyzes and discusses its complexity solution. Based on the analysis of express logistics optimization problem and solution to the complexity, the integrated framework for solving the express logistics optimization problem is proposed, and the double-layer optimized scheme is obtained through effective integration of schemes. A sort of improved ant colony algorithm is chosen for calculated the solution, and the results demonstrate that the model have effective and beneficial influence on improving the management of express.

\section{REFERENCES}

[1] Aksen, D. and O. Kaya, et al. (2012). "Selective and periodic inventory routing problem for waste vegetable oil collection." Optimization Letters 6 (6SI): 1063-1080.

[2] Andersson, H. and A. Hoff, et al. (2010). "Industrial aspects and literature survey: Combined inventory management and routing." Computers \& Operations Research 37 (9): 1515-1536.

[3] Bean, J. C. (1994). "Genetic algorithms and random keys for sequencing and optimization." ORSA Journal on Computing 6 (2): 154-160.

[4] Chien, T. W. and A. Balakrishnan, et al. (1989). "An integrated inventory allocation and vehicle routing problem." Transportation Science 23 (2):6776.

[5] Coelho, L. C. and J. Cordeau, et al. (2012). "The inventory-routing problem with transshipment." Computers \& Operations Research 39 (11): 2537 2548.

[6] Forma, I. and T. Raviv, et al. (2008). "A model for planning of production, inventory and combined routing of delivery and pick-up." International Conference on Industrial Logistics (ICIL 2008): Logistics in a Flat World: Strategy, Management and Operations: 140-151.

[7] Gallego, G. and D. Simchi-Levi (1990). "On the effectiveness of direct shipping strategy for the one-warehouse multi-retailer R-systems." Management Science 36 (2): 240-243.

[8] Moin, N. H. and S. Salhi (2007). "Inventory routing problems: a logistical overview." Journal of the Operational Research Society 58 (9): 1185-1194.

[9] Qiu-Hong, Z. and C. Shuang, et al. (2008). "Model and algorithm for inventory/routing decision in a three-echelon logistics system." European Journal of Operational Research 191 (3): 623-635.

[10] Speranza, M. G. and W. Ukovich (1994). "Minimizing Transportation and Inventory Costs For Several Products on a Single Link." Operations Research 42 (5): 879-894.

[11] Viswanathan, S. and K. Mathur (1997). "Integrating routing and inventory decisions in one-warehouse multiretailer multiproduct distribution systems." Management Science 43 (3): 294-312.

[12] Wan, F. and Q. Zhang (2008). "The research on the inventory routing problem with split pick-ups based on genetic algorithm." 2008 4th International Conference on Wireless Communications, Networking andMobile Computing, VOLS 1-31: 6568-6571.

[13] Yu, Y. and H. Chen, et al. (2008). "A new model and hybrid approach for large scale inventory routing problems." European Journal of Operational Research 189 (3): 1022-1040.

[14] J. Kennedy, R. Eberhart. Particle swarm optimization, IEEE International Conference on Neural Networks, Piscataway: IEEE Press, 1995, pp. 19421948.

[15] A. Azzawi, M. Al-Saedi. Face recognition based on mixed between selected feature by multi-wavelet and particle swarm optimization, in proceedings of Developments in E-systems Engineering (DESE). Piscataway: IEEE Press, 2010, pp. 199-204.

\section{Creative Commons Attribution License 4.0 (Attribution 4.0 International, CC BY 4.0)}

This article is published under the terms of the Creative Commons Attribution License 4.0

https://creativecommons.org/licenses/by/4.0/deed.en US 